\title{
Glucose-responsive oral insulin delivery for postprandial glycemic regulation
}

\author{
Jicheng Yu ${ }^{1}$, Yuqi Zhang ${ }^{1}$, Jinqiang Wang ${ }^{1,2}$, Di Wen ${ }^{1,2}$, Anna R. Kahkoska ${ }^{3}$, John B. Buse ${ }^{3}$, and Zhen Gu ${ }^{1,2,3,4}(\bowtie)$ \\ ${ }^{1}$ Joint Department of Biomedical Engineering, University of North Carolina at Chapel Hill and North Carolina State University, Raleigh, NC 27695, USA \\ ${ }^{2}$ Department of Bioengineering, University of California, Los Angeles, Los Angeles, CA 90095, USA \\ ${ }^{3}$ Department of Medicine, University of North Carolina at Chapel Hill, Chapel Hill, NC 27599, USA \\ ${ }^{4}$ California NanoSystems Institute (CNSI), Jonsson Comprehensive Cancer Center, Center for Minimally Invasive Therapeutics, University of California, Los \\ Angeles, Los Angeles, CA 90095, USA
}

(C) Tsinghua University Press and Springer-Verlag GmbH Germany, part of Springer Nature 2018

Received: 12 October 2018 / Revised: 3 December 2018 / Accepted: 4 December 2018

\begin{abstract}
Controlling postprandial glucose levels for diabetic patients is critical to achieve the tight glycemic control that decreases the risk for developing long-term micro- and macrovascular complications. Herein, we report a glucose-responsive oral insulin delivery system based on Fc receptor (FcRn)-targeted liposomes with glucose-sensitive hyaluronic acid (HA) shell for postprandial glycemic regulation. After oral administration, the HA shell can quickly detach in the presence of increasing intestinal glucose concentration due to the competitive binding of glucose with the phenylboronic acid groups conjugated with HA. The exposed Fc groups on the surface of liposomes then facilitate enhanced intestinal absorption in an FcRn-mediated transport pathway. In vivo studies on chemically-induced type 1 diabetic mice show this oral glucose-responsive delivery approach can effectively reduce postprandial blood glucose excursions. This work is the first demonstration of an oral insulin delivery system directly triggered by increasing postprandial glucose concentrations in the intestine to provide an on-demand insulin release with ease of administration.
\end{abstract}

\section{KEYWORDS}

diabetes, drug delivery, glucose-responsive, insulin, nanomedicine

\section{Introduction}

The worldwide increase in the prevalence of diabetes mellitus is a serious public health burden $[1,2]$. The medical management of diabetes centers around achieving tight control of blood glucose levels to prevent the long-term organ damage that is associated with chronic hyperglycemia [3]. To this end, patients self-administer insulin in a daily regimen that commonly involves subcutaneous injection of commercial insulin formulations [4]. For example, rapid-acting insulin is dosed according to carbohydrate content and subcutaneously injected just before or after each meal to mitigate postprandial glycemic excursions [3]. However, this self-dosing and injection of insulin is associated with a high degree of human error and poor patient compliance, as well as complications such as pain, tissue invasion, infection, and nerve damage $[2,5,6]$. To improve quality of life for patients with diabetes, recent efforts in the past decades have focused on developing alternative strategies based on oral, nasal, pulmonary, and transdermal delivery routes [7-13]. Oral insulin delivery has emerged as one of the most convenient administration routes $[9,14-16]$, and numerous insulin formulations have been developed to enhance oral insulin delivery efficiency by improving intestinal absorption and preventing insulin digestion [17-20]. However, current formulations cannot address the need to release of insulin specifically following meals, where an optimized oral insulin delivery system would supply insulin to the body at the time when it is needed.

In this study, a glucose-responsive liposome-based strategy was developed for oral insulin delivery. This insulin delivery system is composed of a glucose-responsive phenylboronic acid conjugated hyaluronic acid (HA-PBA) shell and a neonatal Fc receptor (FcRn)targeted liposome core loaded with insulin (Fig. 1(a)). FcRn is expressed in the apical region of epithelial cells in the small intestine,

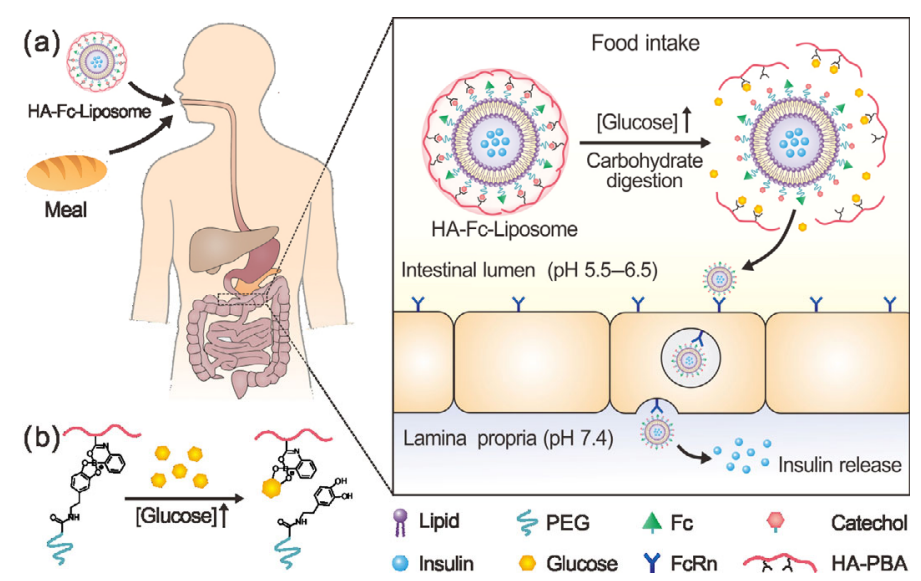

Figure 1 Schematic of the glucose-responsive oral insulin delivery system with glucose-sensitive HA shell for postprandial glycemic regulation. (a) Schematic of insulin-loaded liposomes with glucose-responsive detachable HA shell for oral insulin delivery triggered by an evaluated glucose concentration in the intestine after meals. (b) Schematic of formation and response mechanism of glucoseresponsive HA shell. 
which can bind IgG via the Fc portion, thereby facilitating protein transport across the intestinal epithelium into the circulation [21]. This specific binding functions in a $\mathrm{pH}$-dependent manner, showing high affinity in the apical side ( $\mathrm{pH}$ 5.5-6.5) of the duodenal enterocytes but not in the basolateral side ( $\mathrm{pH} 7.4)[20,22,23]$. Herein, we choose human IgG Fc fragment to modify the surface of the liposomes for FcRn-mediated transepithelial transport [20, 22, 24]. The PBA conjugated HA shell, which coats the liposome core through the boronate ester formation between PBA and catechol groups $[25,26]$ on the liposome surface, can prevent the leakage and digestion of insulin in the gastrointestinal tract, and additionally serves as a glucose-responsive moiety for on-demand insulin absorption. Under elevated postprandial glucose concentrations in the intestine that occurs during meal digestion, the HA shell detaches due to the competitive binding of glucose with PBA (Fig. 1(b)) [27, 28 ], exposing the $\mathrm{Fc}$ groups which promote the intestinal absorption of insulin-loaded liposome core to ultimately release insulin in the bloodstream, reducing blood glucose levels for efficient postprandial glycemic regulation.

\section{Results and discussion}

The insulin-loaded liposomes (Ins-Liposomes) were prepared using the lipid film hydration method $[29,30]$. In order to prepare liposomes with catechol (CA) groups on the surface, dopamine was first conjugated to the carboxyl groups of 1,2-distearoyl-snglycero-3-phosphoethanolamine-N-[carboxy(polyethylene glycol)] (DSPE-PEG-CM) to achieve DSPE-PEG-CA using carbodiimide chemistry. The successful conjugation was verified through UV-Vis spectra (Fig. S1 in the Electronic Supplementary Material (ESM)). The obtained Ins-Liposomes had an average diameter of approximately $69 \mathrm{~nm}$ and a zeta potential of $-2.6 \mathrm{mV}$ (Fig. 2(a)). The encapsulation efficiency (EE) and loading capacity (LC) of insulin in Ins-Liposomes were determined to be $20.7 \%$ and $17.1 \%$, respectively. Polyclonal IgG Fc fragments were covalently conjugated to PEG chain on the surface of Ins-Liposomes using maleimide-thiol chemistry to prepare Fc fragments-modified liposomes (Fc-Liposomes). 2-Iminothiolane was used to modify the Fc with thiol groups [20]. After the conjugation, the hydrodynamic diameter of the Fc-Liposomes increased to $77 \mathrm{~nm}$, and the surface charge showed a minor change to $-6.6 \mathrm{mV}$. The HA shell was further coated on the Fc-Liposome via the combination between PBA and catechol groups [26]. The resulting liposomes with HA shell (HA-Fc-Liposome) had an increased size of around $94 \mathrm{~nm}$ (Fig. 2(b)). The HA-Fc-Liposome showed a significant change in zeta potential from -6.6 to $-28.1 \mathrm{mV}$, indicating the successful formation of HA shell. The transmission electron microscopy (TEM) image revealed the core-shell structure of HA-Fc-Liposomes (Fig. 2(c)). Both the blank HA-Fc-Liposome and Fc-Liposome showed insignificant cytotoxicity towards the human intestinal epithelial colorectal adenocarcinoma (Caco-2) cells (Fig. S2 in the ESM).

The HA shell was expected to not only act as a glucose-responsive moiety, but also prevent the leakage and digestion of insulin in the upper gastrointestinal tract. A quick release of insulin from Fc-Liposomes was observed under $\mathrm{pH} 2.5$ and 7.4 at $37^{\circ} \mathrm{C}$. As shown in Fig. 2(d), around $50 \%$ of insulin was released within $2 \mathrm{~h}$. However, there was negligible leakage of insulin from HA-Fc-Liposomes under the same conditions, which can be attributed to the HA coating. Importantly, insulin levels were nearly completely maintained in the HA-Fc-Liposome under $\mathrm{pH} 2.5$, demonstrating high stability of this insulin carrier in the acidic conditions of the stomach [31].

The HA-PBA was applied to coat the surface of Fc-Liposomes based on the complexation between catechol and PBA through formation of boronate esters [26]. Due to the intramolecular coordination that can stabilize boronate ester formation at a mildly acidic condition (Fig. S3 in the ESM) [25], the boronate ester formation was dynamically stable in the intestine fluid ( $\mathrm{pH} 5.5-8.0)$. However, this kind of boronate ester can be disrupted in the presence of glucose due to competitive binding of glucose with PBA $[26,28]$, thereby rendering the HA shell glucose-responsiveness. To verify the detachment of the HA shell under high glucose concentrations, the HA-Fc-Liposomes containing rhodamine-labelled HA-PBA (Rho-HA-PBA) were incubated in phosphate buffered saline (PBS) buffer with increasing glucose concentrations. As shown in Fig. 3(a), a marked release of Rho-HA-PBA was observed after incubation with $5,10,20$, and $50 \mathrm{mM}$ glucose for $30 \mathrm{~min}$. The morphological change in TEM image and the decrease in size further verified that incubation with $10 \mathrm{mM}$ glucose was sufficient for detachment of the HA shell (Figs. 2(e) and 2(f)). However, insignificant release
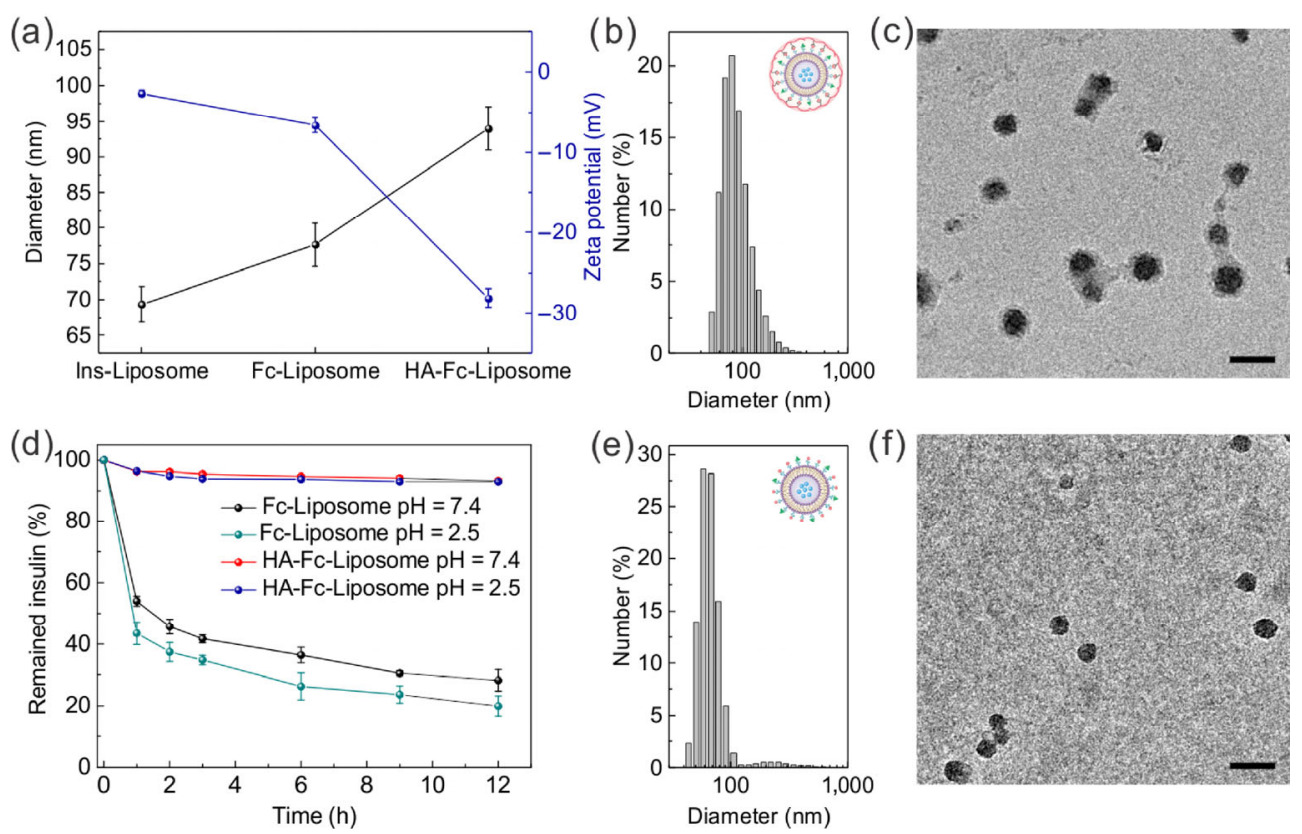

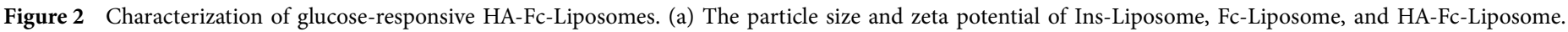

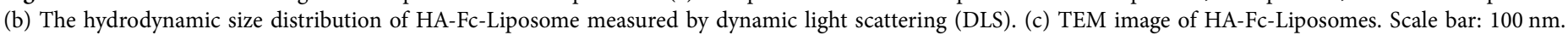

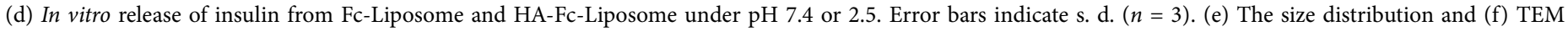
image of HA-Fc-Liposomes after $2 \mathrm{~h}$ incubation with glucose $(10 \mathrm{mM})$. Scale bar is $100 \mathrm{~nm}$. 

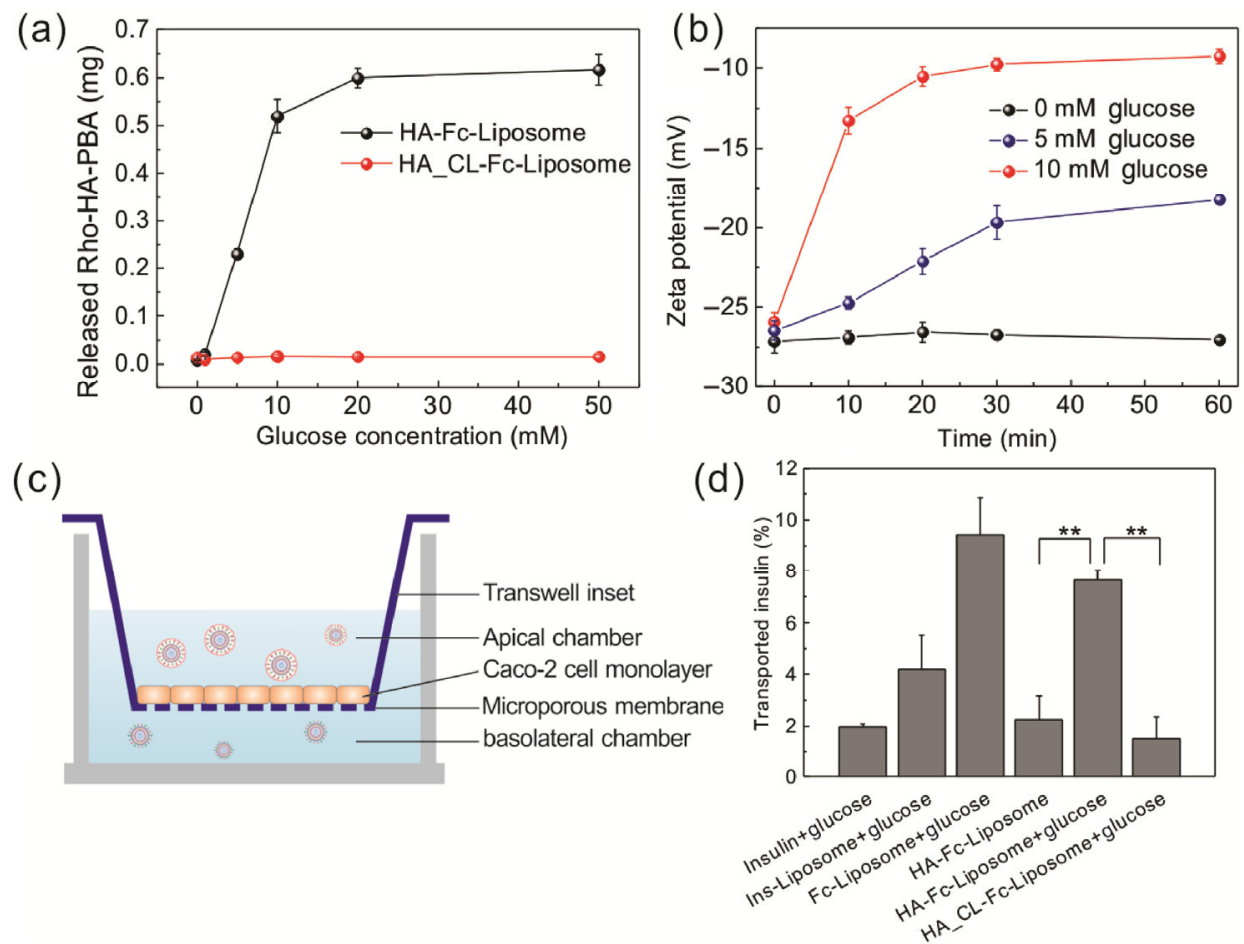

Figure 3 In vitro glucose-triggered HA shell detachment from HA-Fc-Liposome and transepithelial transport. (a) In vitro detachment of Rho-HA-PBA from HA-Fc-Liposome or HA_CL-Fc-Liposome under different glucose concentrations under pH 6.0. Error bars indicate s. d. $(n=3)$. (b) The zeta-potential change of HA-Fc-Liposome in 0, 5, and $10 \mathrm{mM}$ glucose solution over time. Error bars indicate s. d. $(n=3)$. (c) Schematic of in vitro transepithelial transport study using Caco-2 cell monolayer permeability assay. (d) In vitro transepithelial transport of different insulin-loaded liposome formulations with or without $10 \mathrm{mM}$ glucose. Error bars indicate s. d. $(n=3)$. ${ }^{* *} P<0.01$ (two-tailed Student's $t$-test).

was observed from the liposomes coated with crosslinked HA shell (HA_CL-Fc-Liposomes) in all glucose conditions. Zeta potential results further validated the detachment of the HA shell and the subsequent exposure of Fc groups. As shown in Fig. 3(b), the surface charge increased from -25.9 to $-9.7 \mathrm{mV}$ after $30 \mathrm{~min}$ incubation with $10 \mathrm{mM}$ glucose, indicating the quick release of Fc-Liposome core. To determine the effect of the released insulin on blood glucose, insulin-loaded Fc-Liposomes were collected and intravenously injected into the streptozotocin (STZ)-induced adult type 1 diabetic C57BL/6J mice (insulin dose: $5 \mathrm{U} / \mathrm{kg}$ ). Fc-Liposomes were able to generate a hypoglycemic response (Fig. S4 in the ESM) comparable to that of injection of native insulin, confirming the successful release of bioactive insulin.

Since the formation of boronate ester is inhibited in a strong acidic condition in the stomach ( $\mathrm{pH} 1.2-3.0)$, the HA-PBA could not form the complexation with the catechol groups on the surface of the Fc-Liposome core. However, due to the protonation of surface groups on the Fc-Liposomes in acidic conditions, the negative surface charge of Fc-Liposomes was converted to a positive charge under pH 2.5 (Fig. S5 in the ESM), which kept the intact HA shell by electrostatic interaction. Taken together, the HA shell was shown to prevent the leakage and digestion of insulin in the stomach.

In vitro transepithelial transport efficiency of insulin-loaded liposomes was next evaluated using the Caco-2 cell monolayer permeability assay [20,32]. As shown in Fig. 3(c), fluorescein isothiocyanate (FITC)-labelled insulin-loaded liposomes were added to the apical chamber over the cell monolayer and incubated with or without glucose $(10 \mathrm{mM})$ at $37^{\circ} \mathrm{C}$ for $2 \mathrm{~h}$. The transepithelial transport efficiency was determined by measuring the fluorescence intensity of FITC-labelled insulin in the basolateral chamber. Since FcRn is highly expressed on the surface of Caco- 2 cells, higher transepithelial permeability of Fc-Liposomes was observed due to FcRn-mediated transcytosis compared to Ins-liposomes (Fig. 3(d)).
Unlike liposomes with crosslinked HA shell (HA_CL-Fc-Liposomes), the co-incubation with glucose also increased the transport of HAFc-Liposomes, indicating the Fc groups were exposed after glucosetriggered detachment of the HA shell.

We next assessed in vivo performance of glucose-responsive liposomes using STZ-induced type 1 diabetic mice. Immunofluorescence stained sections showed FcRn was expressed in the epithelium of the intestinal villi of the duodenum of STZ-induced diabetic mice (Fig. S6 in the ESM). The diabetic mice were further divided into four groups and sequentially administered by oral gavage: 1) Fc-Liposome + glucose; 2) HA-Fc-Liposome; 3) HA-FcLiposome + glucose; and 4) HA_CL-Fc-Liposome + glucose. Oral intake of glucose solution $(1 \mathrm{~g} / \mathrm{kg})$ post $30 \mathrm{~min}$ oral intake of liposome was used to simulate the postprandial condition [33, 34]. Duodenum sections were collected and imaged $2 \mathrm{~h}$ after administration using fluorescence microscopy. As shown in Fig. 4(a), the green fluorescence of FITC-labelled insulin was ubiquitously observed in the villi from the Fc-Liposome + glucose-treated groups yet rarely observed in the mice treated with HA-Fc-Liposome. Moreover, the additional intake of glucose solution led to the obvious distribution of FITC fluorescence in the villi from the mice treated HA-Fc-Liposome + glucose. There was little fluorescence signal found in the section from HA_CL-Fc-Liposome + glucose-treated mice, as expected. Quantitative data further indicated oral administration of HA-Fc-Liposome + glucose resulted in a 5-fold increase in the amount of FITC-labelled insulin in the villi on the basolateral side of the epithelial cells compared to the HA_CL-Fc-Liposome + glucose-treated group (Fig. 4(b)).

The blood glucose lowering generated by oral administration of insulin-loaded liposomes with or without Fc was assessed on diabetic mice (insulin dose: $10 \mathrm{U} / \mathrm{kg}$ ). Oral intake of Fc-Liposomes induced a significant decline in blood glucose levels during the first $12 \mathrm{~h}$ after treatment (Fig. 4(c)). However, the oral treatment with free 


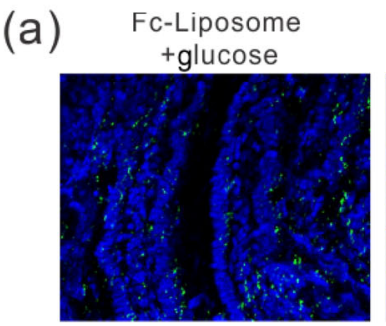

HA-Fc-Liposome

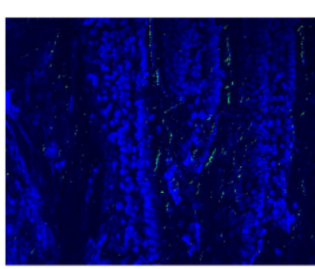

HA-Fc-Liposome + glucose

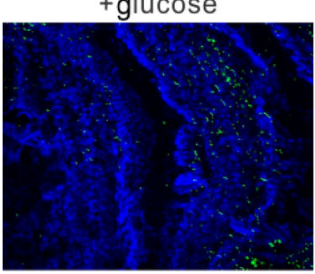

HA_CL-Fc-Liposome + glucose

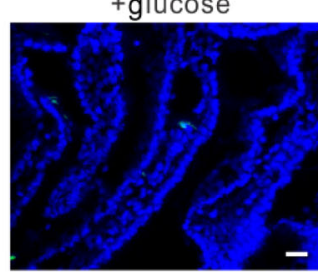

(c) 10

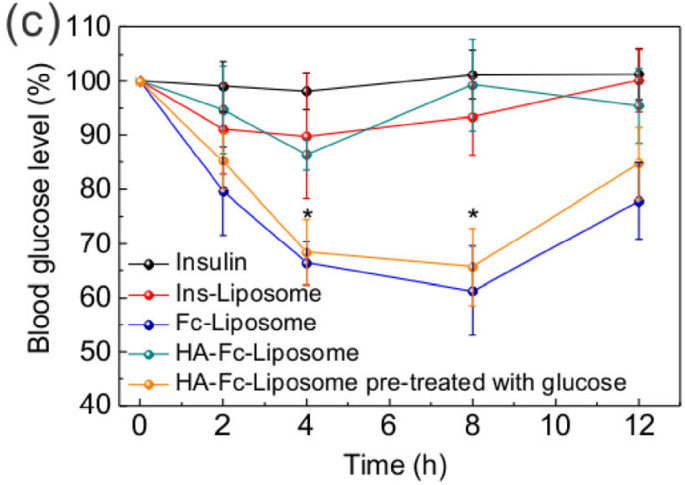

(e)

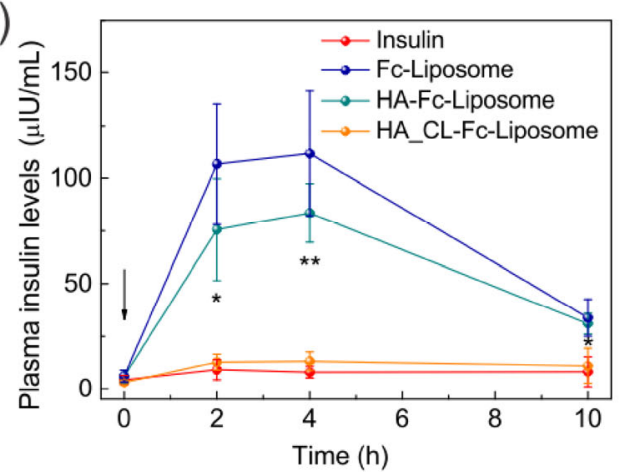

Figure 4 In vivo studies for type 1 diabetes treatment. (a) Fasted STZ-induced type 1 diabetic mice were orally administered with different insulin formulations. Glucose solution $(1 \mathrm{~g} / \mathrm{kg}$ ) was administered post $30 \mathrm{~min}$ oral intake of insulin formulation $(10 \mathrm{U} / \mathrm{kg})$. The intestines were collected for sectioning and imaging $2 \mathrm{~h}$ after administration. Green fluorescence indicated FITC-labelled insulin, and blue fluorescence indicated Hoechst 33342-stained nuclei. Scale bar: $20 \mu \mathrm{m}$. (b) Quantitative analysis of the fluorescence intensities in the in vivo FITC-insulin absorption. All the fluorescence intensities were normalized to the Fc-Liposome + glucose group. Error bars indicate s. d. $(n=3) .{ }^{*} P<0.05$ (two-tailed Student's $t$-test). (c) Blood glucose levels of fasted diabetic mice after oral administration of insulin solution, insulin-loaded Liposome, Fc-Liposome, HA-Fc-Liposome, and HA-Fc-Liposome pre-treated with glucose. Error bars indicate s. d. $(n=5)$. ${ }^{\star} P<0.05$ for administration with HA-Fc-Liposome pre-treated with glucose $(10 \mathrm{mM}$ ) compared with HA-Fc-Liposome. (d) Blood glucose levels of fasted mice orally administered with different insulin formulations: 1) untreated; 2) insulin solution; 3) Fc-Liposome; 4) HA-Fc-Liposome; 5) HA_CL-Fc-Liposome. Glucose solution (1 g/kg) was orally administered post $30 \mathrm{~min}$ oral intake of insulin formulation $\left(10 \mathrm{U} / \mathrm{kg}\right.$ ). (e) Plasma human insulin concentrations in diabetic mice after treatment. ${ }^{\star} P<0.05$ and ${ }^{*} P<$ 0.01 for administration with HA-Fc-Liposome compared with HA_CL-Fc-Liposome. The black arrows indicate the oral administration of glucose solution. Error bars indicate s. d. $(n=5)$.

insulin did not induce a hypoglycemic response, and the treatment of Ins-Liposomes or HA-Fc-Liposomes led to minimal reduction in blood glucose levels. Of note, the oral administration of HA-FcLiposomes that had been previously treated with $10 \mathrm{mM}$ glucose also showed a significant blood glucose lowering effect, validating the exposed Fc improved the intestinal absorption of the liposomes after the glucose-triggered detachment of HA shell.

Next, we assessed the glucose-responsive intestinal absorption of liposomes by oral administration of glucose solution to simulate the postprandial condition. Glucose solution $(1 \mathrm{~g} / \mathrm{kg})$ was orally administered to mice $30 \mathrm{~min}$ after the oral administration of different insulin-loaded formulations. As demonstrated in Fig. 4(d), oral administration of glucose solution led to postprandial hyperglycemia, and the blood glucose levels reduced to the initial level after $8 \mathrm{~h}$ of treatment. Pre-treatment of insulin-loaded Fc-Liposomes significantly suppressed the postprandial elevation of blood glucose, where blood glucose levels returned to initial levels after $2 \mathrm{~h}$ of oral glucose treatment and remained at such reduced levels for $8 \mathrm{~h}$. Treatment with glucose-responsive HA-Fc-Liposomes similarly exhibited a glucose-lowering effect due to the Fc-mediated intestinal absorption of insulin-loaded liposomes after the detachment of HA shell, triggered by the high glucose concentration in the intestine after oral glucose administration. However, a significant lasting postprandial increase of blood glucose was observed in the group administered HA_CLFc-Liposome, which further verified the blood glucose lowering action of this insulin delivery system was dependent on the intestinal glucose-triggered detachment of HA shell. Correspondingly, mice treated with HA-Fc-Liposomes presented a consistently higher plasma insulin concentration than those treated with HA_CL-Fc-Liposome (Fig. 4(e)).

\section{Conclusion}

Postprandial glycemic control is critical to prevent microvascular and macrovascular complications and maintain insulin resistance in people with diabetes [35]. Current management strategies to control 
postprandial glucose levels involve quantifying carbohydrates [36] to calculate insulin dose [37] and multiple injections of fast-acting insulin before or with a meal for type 1 and advanced type 2 diabetic patients; yet these methods are inaccurate and time-consuming, requiring consideration of carbohydrates quantity and distribution at each meal and snack. There remains a clear need to optimize the efficacy of prandial insulin dosing to confer the greatest protection from diabetic complications.

In this work, we exploited an FcRn-targeted insulin-loaded liposome with a glucose-responsive detachable HA shell for postprandial glycemic control. The increased intestinal glucose concentration that occurs with digestion of a carbohydrate-containing meal was utilized to trigger the detachment of HA shell and subsequent exposure of Fc for enhancing the intestinal absorption by FcRnmediated transepithelial transport. Thorough in vivo studies demonstrated that this "smart insulin pill" was able to regulate blood glucose levels in diabetic mice after oral intake of glucose solution simulating a meal. Furthermore, the single administration of HA-Fc-Liposomes did not reduce the blood glucose levels under fasting conditions, thereby effectively preventing the risk of hypoglycemia. In addition, it was reported that FcRn expression in the intestine of rodents is significantly down-regulated after weaning, while FcRn is expressed within the adult human intestine throughout life [38]. Therefore, the transepithelial transport for potential human use will be more efficient. This "food-responsive" formulation strategy integrating an intestinal signal trigger also has clinical potential in the oral delivery of other therapeutic agents for treating a variety of metabolic diseases $[31,39]$.

\section{Experimental}

\subsection{Materials}

All chemicals were purchased from Sigma-Aldrich unless otherwise specified and were used as received. Sodium hyaluronic acid was purchased from Freda Biochem Co., Ltd. (Shandong, China). Human recombinant insulin (Zn salt, $27.5 \mathrm{IU} / \mathrm{mg}$ ) was purchased from Life Technology. Purified human polyclonal IgG Fc was purchased from Bethyl Laboratories. 1,2-Distearoyl-sn-glycero-3phosphoethanolamine-N-[maleimide(polyethylene glycol)] (DSPEPEG-MAL) $\left(M_{\mathrm{W}}=2,000\right)$ and DSPE-PEG-CM $\left(M_{\mathrm{W}}=2,000\right)$ were purchased from Laysan Bio, Inc.

\subsection{Synthesis and characterization of glucose-responsive HA-PBA, acrylate modified HA-PBA, and rhodamine-labelled HA-PBA}

Acrylate modified HA and rhodamine-labelled HA were synthesized following Refs. [40, 41]. 2-Aminophenylboronic was conjugated to HA in the presence of 1-ethyl-3-(3-dimethylaminopropyl)carbodiimide hydrochloride (EDC) and N-hydroxysuccinimide (NHS). Briefly, $0.5 \mathrm{~g}$ of HA (molecular weight: $10 \mathrm{kDa}$ ) was dissolved in water, to which EDC (0.58 g) and NHS (0.35 g) were added and stirred for $15 \mathrm{~min}$ at room temperature (RT). Then 2-aminophenylboronic acid hydrochloride $(0.17 \mathrm{~g})$ was added to the mixture and reacted at RT overnight. The reaction solution was thoroughly dialyzed against deionized (DI) water for 3 days. Then, HA-PBA was obtained by lyophilization and characterized by ${ }^{1} \mathrm{H}$ NMR. The degree of modification was calculated to be $14.4 \%$ by comparing the ratio of the areas under the proton peaks at 6.91-7.40 ppm to the peak at $1.99 \mathrm{ppm} .{ }^{1} \mathrm{H}$ NMR (400 MHz, $\left.\mathrm{D}_{2} \mathrm{O}, \delta\right): 7.40(\mathrm{~s}, \mathrm{H}$, phenyl-H), 7.20 (s, 2H, phenyl-H), 6.91 (s, H, phenyl-H). Acrylate modified HA-PBA and rhodamine-labelled HA-PBA were synthesized in a similar protocol.

\subsection{Synthesis and characterization of DSPE-PEG-CA}

DSPE-PEG-CM (200 mg), EDC (57.5 mg), and NHS (34.5 mg) were mixed and dissolved in $20 \mathrm{~mL}$ DI water and stirred for $30 \mathrm{~min}$ at RT. Dopamine hydrochloride $(57 \mathrm{mg}$ ) was then added to the mixture and stirred for $8 \mathrm{~h}$ at RT. The unreacted dopamine was removed by dialysis against DI water for 2 days. The obtained DSPE-PEG-CA was lyophilized and stored at $4{ }^{\circ} \mathrm{C}$ until use. The successful conjugation was verified by measuring the UV-Vis absorption of catechol groups using a Nanodrop 2000C spectrometer (Thermo Scientific).

\subsection{Preparation of glucose-responsive liposomes loaded with insulin}

First, the insulin-loaded liposome was prepared by the lipid film hydration method. Briefly, a mixture of egg phosphatidylcholine (EPC), dioleoylphosphatidylethanolamine (DOPE), DSPE-PEG-MAL, DSPE-PEG-CA and cholesterol (weight ratio = 9:9:1:1:4) was dissolved in chloroform. The solution was evaporated dry to form the lipid film. The formed lipid film was hydrated with 4-(2hydroxyethyl)-1-piperazineethanesulfonic acid (HEPES) buffer ( $5 \mathrm{mM}$ ) containing insulin (insulin:lipids, 1:1, w:w), dispersed by a probe-type ultrasonicator, and extruded 3 times through the membrane filters with the pore size of 0.45 and $0.20 \mu \mathrm{m}$ successively. The nonencapsulated insulin was removed by centrifugation at $21,000 \mathrm{rpm}$ and the resulting Ins-Liposomes were washed 3 times by PBS buffer containing $5 \mathrm{mM}$ ethylene diamine tetraacetic acid (EDTA). The EE and LC of insulin in Ins-Liposomes were determined by measuring the amount of non-encapsulated insulin using a Coomassie Plus protein assay.

Fc-Liposomes were obtained by conjugation of Fc to PEG chain on the surface of Ins-Liposomes using maleimide-thiol chemistry. Briefly, $86 \mu \mathrm{g}$ of purified human polyclonal IgG Fc in PBS containing $5 \mathrm{mM}$ EDTA was reacted with $4.8 \mu \mathrm{L}$ of Traut's Reagent $(0.5 \mathrm{mg} / \mathrm{mL})$ for $1 \mathrm{~h}$. The modified Fc was then added to the Ins-Liposomes and mixed for $1 \mathrm{~h}$ at $4{ }^{\circ} \mathrm{C}$. The resulting Fc-Liposomes were collected by centrifugation at 21,000 rpm and washed with PBS buffer 3 times.

The obtained Fc-Liposome solution was added to the HA-PBA solution (HA-PBA:lipids, 1:10, w:w) and mixed for $2 \mathrm{~h}$. The HA-FcLiposomes were obtained by centrifugation at $21,000 \mathrm{rpm}$ and washed with PBS buffer. The zeta potential and size distribution were measured on the Zetasizer (Nano ZS, Malvern). The TEM images of HA-Fc-Lipo were obtained on a JEOL 2000FX TEM instrument.

The liposomes with non-glucose-responsive HA shell (HA_CLFc-Liposomes) were prepared by crosslinking HA shell via UV irradiation. The Fc-Liposomes were added into the acrylate modified HA-PBA solution (HA-PBA:lipids, 1:10, w:w) and stirred for $2 \mathrm{~h}$. A crosslinker N,N-methylenebisacrylamide (MBA) (MBA:HA-PBA, 1:5, w:w) and a photo-initiator Irgacure $2959(0.1 \%, \mathrm{w}: \mathrm{v})$ was then added to the mixture. After radical polymerization via UV radiation for $60 \mathrm{~s}$ using a BlueWave 75 UV Curing Spot Lamp (DYMAX), HA_CL-Fc-Liposomes were obtained by centrifugation at 21,000 rpm and washed with PBS buffer.

\subsection{In vitro detachment of HA shell}

To evaluate the glucose-responsive detachment of HA-PBA shell, HA-Fc-Liposomes or HA_CL-Fc-Liposomes containing rhodaminelabelled HA-PBA were incubated under $\mathrm{pH} 6.0$ with different glucose concentrations $(1,5,10,20$, and $50 \mathrm{mM})$ at $37^{\circ} \mathrm{C}$ for $1 \mathrm{~h}$. The detached Rho-HA-PBA were separated by centrifugation at 21,000 rpm. The fluorescence intensity of free Rho-HA-PBA was determined at $575 \mathrm{~nm}$ with an excitation wavelength of $552 \mathrm{~nm}$ by a microplate reader (Infinite M200 PRO, Tecan).

\subsection{In vitro insulin release}

To evaluate the in vitro insulin release profile, FITC-insulin-loaded $\mathrm{HA}-\mathrm{Fc}$-Liposomes or Fc-Liposomes were suspended in Tris- $\mathrm{HCl}$ buffer (10 mM, pH 7.4) or Tris- $\mathrm{HCl}$ buffer (10 mM, pH 2.5). 
At prearranged time intervals, released insulin was harvested by centrifugation at 21,000 rpm. The fluorescence intensity of free FITCinsulin was determined at $519 \mathrm{~nm}$ with the excitation wavelength at $495 \mathrm{~nm}$.

\subsection{Cell culture}

Caco-2 cells were obtained from Tissue Culture Facility of UNC Lineberger Comprehensive Cancer Center and cultured in Dulbecco's Modified Eagle's Medium supplemented with $10 \%$ (v/v) fetal bovine serum (FBS), penicillin $(100 \mathrm{U} / \mathrm{mL})$ and streptomycin $(100 \mu \mathrm{g} / \mathrm{mL})$ in a $37{ }^{\circ} \mathrm{C}$ incubator (Thermal Scientific) under $5 \% \mathrm{CO}_{2}$ and $90 \%$ humidity. The cells were regularly sub-cultured with trypsin-EDTA $(0.25 \%, w / w)$ and cell density was determined with hemocytometer before each experiment.

\subsection{In vitro cytotoxicity}

The cytotoxicity of the HA-Fc-Liposomes and Fc-Liposomes were evaluated using 3-(4,5)-dimethylthiahiazo(-z-y1)-3,5-diphenytetrazoliumromide (MTT) assay with Caco-2 cells. Prior to the test, the medium in 96-well plate was removed. After washing with PBS, serial dilutions of bare HA-Fc-Liposomes or Fc-Liposomes were added into wells. After $24 \mathrm{~h}$ incubation, thiazolyl blue solution $(5 \mathrm{mg} / \mathrm{mL})$ was added to each well and incubated for another $4 \mathrm{~h}$. After removing the medium, the purple formazan crystal was dissolved in $150 \mu \mathrm{L}$ of dimethyl sulfoxide (DMSO). The absorbance of the plates was read at $570 \mathrm{~nm}$ by a microplate reader.

\subsection{In vitro transcytosis studies}

To further investigate the transport of insulin-loaded liposomes across the epithelial cells, the Caco- 2 cell monolayer was incubated for 21 days after being seeded on the Transwell plates (Costar). Prior to the studies, the medium was replaced with pre-warmed Hanks' balanced salt solution (HBSS) ( $\mathrm{pH} \mathrm{6.0)} \mathrm{in} \mathrm{the} \mathrm{apical} \mathrm{chamber} \mathrm{and}$ HBSS ( $\mathrm{pH} 7.4$ ) in the basolateral chamber and allowed to equilibrate for $1 \mathrm{~h}$ at $37^{\circ} \mathrm{C}$. Afterwards, the apical solution was replaced with $200 \mu \mathrm{L}$ of FITC-insulin + glucose $(10 \mathrm{mM})$, Ins-Liposomes + glucose $(10 \mathrm{mM})$, Fc-Liposomes + glucose $(10 \mathrm{mM})$, HA-Fc-Liposomes, HA-Fc-Liposomes + glucose $(10 \mathrm{mM})$, and HA_CL-Fc-Liposomes + glucose $(10 \mathrm{mM})$, respectively. After incubation for $2 \mathrm{~h}$ at $37^{\circ} \mathrm{C}$, the basolateral solution was collected and the amount of transported FITC-insulin was determined using a microplate reader at $519 \mathrm{~nm}$ with the excitation wavelength at $495 \mathrm{~nm}$.

\subsection{Immunofluorescence staining}

Small intestine was harvested and fixed in $10 \%$ formalin. Then the tissues were embedded and cut into $8 \mu \mathrm{M}$ thick sections. After rehydration, the slides were blocked and incubated with IgG or anti-mouse FcRn primary antibodies (Sigma Cat No. ABF191) overnight and then incubated with anti-rabbit secondary antibodies. The expression of FcRn was detected under fluorescence microscope.

\subsection{In vivo absorption}

The animal study protocol was approved by the Institutional Animal Care and Use Committee at North Carolina State University and University of North Carolina at Chapel Hill. STZ-induced adult diabetic mice (male C57B6, Jackson Lab, USA) were fasted for $6 \mathrm{~h}$ before administration. The mice were administered with the following formulations by oral gavage: Fc-Liposomes, HA-Fc-Liposomes, and HA_CL-Fc-Liposomes $(4 \mathrm{~mL} / \mathrm{kg})$. After $30 \mathrm{~min}$, the experiment group was further orally administered glucose solution $(1 \mathrm{~g} / \mathrm{kg})$. The mice were euthanized and the duodenum tissue were collected for frozen section after $2 \mathrm{~h}$. Hoechst 33342 was used for nuclei staining, and the cross sections of the tissue were observed by fluorescence microscope.

\subsection{In vivo studies using STZ-induced diabetic mice}

The in vivo efficacy of glucose-responsive insulin-loaded liposome for diabetes treatment was evaluated on STZ-induced adult diabetic mice (male C57B6, Jackson Lab, USA). Mouse glucose levels were monitored for two days before administration, and all mice were fasted for $6 \mathrm{~h}$ before administration. Mice $(n=5)$ were chosen per group such that the mean initial blood glucose levels were between $300-500 \mathrm{mg} / \mathrm{dL}$ per group. Before the administration, HA-FcLiposomes were incubated under $\mathrm{pH} 6.0$ with $10 \mathrm{mM}$ glucose at $37^{\circ} \mathrm{C}$ for $1 \mathrm{~h}$. The pre-treated HA-Fc-Liposomes were collected by centrifugation at $21,000 \mathrm{rpm}$. The mice were administered with the following formulations by oral gavage: insulin solution, Ins-Lipsomes, Fc-Liposomes, HA-Fc-Liposomes, and HA-Fc-Liposomes pre-treated with glucose (insulin dose: $10 \mathrm{U} / \mathrm{kg}$ ).

In order to assess the glucose-responsive intestinal absorption of liposomes by oral administration of glucose solution to simulate the postprandial condition, the mice were administered with the following formulations by oral gavage: insulin solution, Fc-Liposomes, HAFc-Liposomes, and HA_CL-Fc-Liposomes (insulin dose: $10 \mathrm{U} / \mathrm{kg}$ ). After $30 \mathrm{~min}$, the experiment group was further orally administered glucose solution $(1 \mathrm{~g} / \mathrm{kg})$. The glucose levels of each mouse were monitored over time using the Clarity GL2Plus glucose meter (Clarity Diagnostics, Boca Raton, Florida, USA). The plasma insulin concentration was measured using Invitrogen ${ }^{\mathrm{TM}}$ Novex $^{\mathrm{TM}}$ EASIA $^{\mathrm{TM}}$ Insulin Human ELISA Kit.

\subsection{Statistical analysis}

All results presented are Mean $\pm \mathrm{s}$. d. Statistical analysis was performed using two-tailed Student's $t$-tests. ${ }^{\star} P$ value $<0.05$ describing the differences between experimental groups and control groups was considered statistically significant.

\section{Acknowledgements}

This work was supported by the grants from NC TraCS, NIH's Clinical and Translational Science Awards (CTSA, NIH grant 1UL1TR001111) at UNC-CH and Sloan Research Fellowship. We acknowledge the use of the Analytical Instrumentation Facility (AIF) at NC State, which is supported by the State of North Carolina and the National Science Foundation (NSF).

\section{Competing Interests}

Z. G. and J. C. Y. have applied for patents related to this study. Z. G. is a scientific co-founder of Zenomics Inc.

Electronic Supplementary Material: Supplementary material (UV-Vis spectra, cytotoxicity assay results, equilibrium mechanism of HA-PBA in water, in vivo bioactivity, zeta potential, and FcRn expression) is available in the online version of this article at https://doi.org/10.1007/s12274-018-2264-9.

\section{References}

[1] Mo, R.; Jiang, T. Y.; Di, J.; Tai, W. Y.; Gu, Z. Emerging micro- and nanotechnology based synthetic approaches for insulin delivery. Chem. Soc. Rev. 2014, 43, 3595-3629.

[2] Veiseh, O.; Tang, B. C.; Whitehead, K. A.; Anderson, D. G.; Langer, R. Managing diabetes with nanomedicine: Challenges and opportunities. Nat. Rev. Drug Discov. 2015, 14, 45-57.

[3] Owens, D. R.; Zinman, B.; Bolli, G. B. Insulins today and beyond. Lancet 2001, 358, 739-746.

[4] Owens, D. R. New horizons-Alternative routes for insulin therapy. Nat. Rev. Drug Discov. 2002, 1, 529-540.

[5] Bratlie, K. M.; York, R. L.; Invernale, M. A.; Langer, R.; Anderson, D. G. Materials for diabetes therapeutics. Adv. Healthc. Mater. 2012, 1, 267-284. 
[6] Ravaine, V.; Ancla, C.; Catargi, B. Chemically controlled closed-loop insulin delivery. J. Control. Release 2008, 132, 2-11.

[7] Heinemann, L.; Pfutzner, A.; Heise, T. Alternative routes of administration as an approach to improve insulin therapy: Update on dermal, oral, nasal and pulmonary insulin delivery. Curr. Pharm. Des. 2001, 7, 1327-1351.

[8] Owens, D. R.; Zinman, B.; Bolli, G. Alternative routes of insulin delivery. Diabet. Med. 2003, 20, 886-898.

[9] Carino, G. P.; Mathiowitz, E. Oral insulin delivery. Adv. Drug Deliv. Rev. 1999, 35, 249-257.

[10] Cefalu, W. T. Concept, strategies, and feasibility of noninvasive insulin delivery. Diabetes Care 2004, 27, 239-246.

[11] Yu, J. C.; Zhang, Y. Q.; Ye, Y. Q.; DiSanto, R.; Sun, W. J.; Ranson, D.; Ligler, F. S.; Buse, J. B.; Gu, Z. Microneedle-array patches loaded with hypoxia-sensitive vesicles provide fast glucose-responsive insulin delivery. Proc. Natl. Acad. Sci. USA 2015, 112, 8260-8265.

[12] Yu, J. C.; Zhang, Y. Q.; Bomba, H.; Gu, Z. Stimuli-responsive delivery of therapeutics for diabetes treatment. Bioeng. Transl. Med. 2016, 1, 323-337.

[13] Makino, K.; Mack, E. J.; Okano, T.; Kim, S. W. A microcapsule selfregulating delivery system for insulin. J. Control. Release 1990, 12, 235-239.

[14] Iyer, H.; Khedkar, A.; Verma, M. Oral insulin-A review of current status. Diabetes Obes. Metab. 2010, 12, 179-185.

[15] Mitragotri, S.; Burke, P. A.; Langer, R. Overcoming the challenges in administering biopharmaceuticals: Formulation and delivery strategies. Nat. Rev. Drug Discov. 2014, 13, 655-672.

[16] Moroz, E.; Matoori, S.; Leroux, J. C. Oral delivery of macromolecular drugs: Where we are after almost 100 years of attempts. Adv. Drug Deliv. Rev. 2016, 101, 108-121.

[17] Lowman, A. M.; Morishita, M.; Kajita, M.; Nagai, T.; Peppas, N. A. Oral delivery of insulin using pH-responsive complexation gels. J. Pharm. Sci. 1999, 88, 933-937.

[18] Sonaje, K.; Lin, K. J.; Wang, J. J.; Mi, F. L.; Chen, C. T.; Juang, J. H.; Sung, H. W. Self-assembled $\mathrm{pH}$-sensitive nanoparticles: A platform for oral delivery of protein drugs. Adv. Funct. Mater. 2010, 20, 3695-3700.

[19] Yin, L. C.; Ding, J. Y.; He, C. B.; Cui, L. M.; Tang, C.; Yin, C. H. Drug permeability and mucoadhesion properties of thiolated trimethyl chitosan nanoparticles in oral insulin delivery. Biomaterials 2009, 30, 5691-5700.

[20] Pridgen, E. M.; Alexis, F.; Kuo, T. T.; Levy-Nissenbaum, E.; Karnik, R.; Blumberg, R. S.; Langer, R.; Farokhzad, O. C. Transepithelial transport of Fc-targeted nanoparticles by the neonatal fc receptor for oral delivery. Sci. Transl. Med. 2013, 5, 213 ra167.

[21] Raghavan, M.; Gastinel, L. N.; Bjorkman, P. J. The class I major histocompatibility complex related $\mathrm{Fc}$ receptor shows $\mathrm{pH}$-dependent stability differences correlating with immunoglobulin binding and release. Biochemistry 1993, 32, 8654-8660.

[22] Qin, J. J.; Wang, W.; Sarkar, S.; Zhang, R. W. Oral delivery of anti-MDM2 inhibitor SP141-loaded FcRn-targeted nanoparticles to treat breast cancer and metastasis. J. Control. Release 2016, 237, 101-114.

[23] He, W. Z.; Ladinsky, M. S.; Huey-Tubman, K. E.; Jensen, G. J.; McIntosh, J. R.; Björkman, P. J. FcRn-mediated antibody transport across epithelial cells revealed by electron tomography. Nature 2008, 455, 542-546.

[24] Shi, Y. N.; Sun, X. F.; Zhang, L. P.; Sun, K. X.; Li, K. K.; Li, Y. X.; Zhang, Q. Fc-modified exenatide-loaded nanoparticles for oral delivery to improve hypoglycemic effects in mice. Sci. Rep. 2018, 8, 726.

[25] Deng, C. C.; Brooks, W. L. A.; Abboud, K. A.; Sumerlin, B. S. Boronic acid-based hydrogels undergo self-healing at neutral and acidic pH. ACS Macro Lett. 2015, 4, 220-224.

[26] Brooks, W. L. A.; Sumerlin, B. S. Synthesis and applications of boronic acid-containing polymers: From materials to medicine. Chem. Rev. 2015, $116,1375-1397$.

[27] Kataoka, K.; Miyazaki, H.; Bunya, M.; Okano, T.; Sakurai, Y. Totally synthetic polymer gels responding to external glucose concentration: Their preparation and application to on-off regulation of insulin release. J. Am. Chem. Soc. 1998, 120, 12694-12695.

[28] Chou, D. H. C.; Webber, M. J.; Tang, B. C.; Lin, A. B.; Thapa, L. S.; Deng, D.; Truong, J. V.; Cortinas, A. B.; Langer, R.; Anderson, D. G. Glucose-responsive insulin activity by covalent modification with aliphatic phenylboronic acid conjugates. Proc. Natl. Acad. Sci. USA 2015, 112, 2401-2406.

[29] Mo, R.; Jiang, T. Y.; Gu, Z. Enhanced anticancer efficacy by ATP-mediated liposomal drug delivery. Angew. Chem., Int. Ed. 2014, 53, 5815-5820.

[30] Zhan, C. Y.; Wang, W. P.; Santamaria, C.; Wang, B.; Rwei, A.; Timko, B. P.; Kohane, D. S. Ultrasensitive phototriggered local anesthesia. Nano Lett. 2017, 17, 660-665.

[31] Lu, Y.; Aimetti, A. A.; Langer, R.; Gu, Z. Bioresponsive materials. Nat. Rev. Mater. 2016, 2, 16075.

[32] Jin, Y.; Song, Y. P.; Zhu, X.; Zhou, D.; Chen, C. H.; Zhang, Z. R.; Huang, Y. Goblet cell-targeting nanoparticles for oral insulin delivery and the influence of mucus on insulin transport. Biomaterials 2012, 33, 1573-1582.

[33] Li, Y. H.; Wen, S. P.; Kota, B. P.; Peng, G.; Li, G. Q.; Yamahara, J.; Roufogalis, B. D. Punica granatum flower extract, a potent $\alpha$-glucosidase inhibitor, improves postprandial hyperglycemia in Zucker diabetic fatty rats. J. Ethnopharmacol. 2005, 99, 239-244.

[34] Kim, J. H.; Kang, M. J.; Choi, H. N.; Jeong, S. M.; Lee, Y. M.; Kim, J. I. Quercetin attenuates fasting and postprandial hyperglycemia in animal models of diabetes mellitus. Nutr. Res. Pract. 2011, 5, 107-111.

[35] Bell, K. J.; King, B. R.; Shafat, A.; Smart, C. E. The relationship between carbohydrate and the mealtime insulin dose in type 1 diabetes. J. Diabetes Complications 2015, 29, 1323-1329.

[36] Wolever, T. M.; Bolognesi, C. Source and amount of carbohydrate affect postprandial glucose and insulin in normal subjects. J. Nutr. 1996, 126, 2798-2806.

[37] American Diabetes Association, A. D. Standards of medical care in diabetes-2017: Summary of revisions. Diabetes Care 2017, 40, S4-S5.

[38] Roopenian, D. C.; Akilesh, S. FcRn: The neonatal Fc receptor comes of age. Nat. Rev. Immunol. 2007, 7, 715-725.

[39] Wang, Y. F.; Kohane, D. S. External triggering and triggered targeting strategies for drug delivery. Nat. Rev. Mater. 2017, 2, 17020.

[40] Di, J.; Yu, J. C.; Ye, Y. Q.; Ranson, D.; Jindal, A.; Gu, Z. Engineering synthetic insulin-secreting cells using hyaluronic acid microgels integrated with glucose-responsive nanoparticles. Cell. Mol. Bioeng. 2015, 8, 445-454.

[41] Zhang, Y. Q.; Yu, J. C.; Wang, J. Q.; Hanne, N. J.; Cui, Z.; Qian, C. G.; Wang, C.; Xin, H. L.; Cole, J. H.; Gallippi, C. M. et al. Thrombin-responsive transcutaneous patch for auto-anticoagulant regulation. Adv. Mater. 2017, 29,1604043 . 